\title{
Analysis of Dynamic Characteristics of Spiral-Grooved Journal Bearing with Considering Cavitation Occurrence*
}

\author{
Tomoko HIRAYAMA*, Takeo SAKURAI*** and Hiroshi YABE ${ }^{* * * *}$
}

\begin{abstract}
Dynamic characteristics of an oil-lubricated spiral-grooved journal bearing are theoretically investigated in this paper with considering the effect of cavitation occurrence in the bearing clearance. The Reynolds equation by cooperating with a cavitation theory called 'equivalent flow model' as a formulation for cavitation occurrence is expanded by perturbation scheme with respect to the journal eccentricity, so that the dynamic bearing characteristics, such as bearing stiffnesses and damping coefficients, are calculated from the obtained results of perturbed pressure distributions. It is shown that, when the cavitation occurrence is considered, the value of the direct bearing stiffness becomes larger, while those of the crosscoupling bearing stiffness and damping coefficients become smaller, compared with those with neglecting the contribution of the cavitation occurrence. Stable operating limits against 'half-frequency whirl' are discussed as well, showing that the critical mass values become larger when considering the cavitation occurrence than those with neglecting it.
\end{abstract}

Key Words: Spiral-Grooved Bearing, Journal Bearing, Cavitation Occurrence, Dynamic Bearing Characteristics, Stable Operating Limit, Computational Analysis

\section{Introduction}

Performances of precision equipments such as audiovisual $(\mathrm{AV})$ or office-automation $(\mathrm{OA})$ instruments have made rapid progresses day by day, and improvements of the operating states of their motor spindles have been more strongly demanded than before. For examples, spindles of polygonal mirror motors equipped in laser beam printers are required to rotate as fast as $30000 \mathrm{rpm}$, while those of hard disk drive (HDD) motors are to rotate as accurate as $10 \mathrm{~nm}$ in terms of non-repeatable run-out. With these trends for a background, hydrodynamic fluid film bearings, especially spiral-grooved bearings, are recently applied to the spindles as the alternative for current ball

* Received 4th June, 2004 (No. 02-0732). Japanese Original: Trans. Jpn. Soc. Mech. Eng., Vol.69, No.684, C (2003), pp.2206-2212 (Received 7th June, 2002)

** Department of Mechanical and Systems Engineering, Ryukoku University, 1-5 Yokotani, Seta, Oe-cho, Otsu, Shiga 520-2194, Japan.

E-mail: tomoko@rins.ryukoku.ac.jp

*** Department of Precision Engineering, Kyoto University, Yoshida-honmachi, Sakyo-ku, Kyoto 606-8501, Japan

${ }^{* * * *}$ Department of Mechanical Engineering, Osaka ElectroCommunication University, 18-8 Hatsu-cho, Neyagawa, Osaka 572-8530, Japan. E-mail: yabe@isc.osakac.ac.jp bearings ${ }^{(1)}$. The motive of the alternation may be mainly derived from the fact that fluid film bearings are generally superior to ball bearings from viewpoints of attainable maximum rotational speed and run-out characteristics $^{(2)-(4)}$.

As to hydrodynamic fluid film bearings, it is well known that 'cavitation' generally occurs in negative pressure regions in the bearing clearance. The negative pressure regions are naturally formed when the shaft (or relatively sleeve) is in an eccentric state. To have an exact grasp of characteristics of hydrodynamic fluid film bearings, the effect of cavitation occurrence in the bearing clearance should be examined in the process of theoretical analysis. Though Half-Sommerfeld or Swift-Stieber (Reynolds) condition is commonly applied as cavitation model in the course to solve Reynolds equation, both of them have some serious shortcomings. The former cannot satisfy the continuity condition of fluid flow at the boundary of liquid-gas interface, while the latter necessarily requires a complicated calculation procedure for almost all cases except for a plain journal bearing. The authors, then, focus on the 'equivalent flow model'(5), proposed by Ikeuchi, K., et al., as another cavitation model. In this model, the cavitated fluid flow is treated as a single-phase laminar flow having equivalent density and viscosity, thus, the model leads to a simple formulation of the calculation 
scheme of pressure distribution in the bearing clearance when we take the cavitation occurrence into consideration. It should be also noted that the model can be easily applied even to the complicated bearing configuration cases such as spiral-grooved bearings with exactly satisfying the continuity condition of fluid flow everywhere in the bearing area. Positions and areas of real cavitated regions can be calculated from the obtained pressure distribution based on the model.

Based on those backgrounds, the authors have investigated the behavior of cavitation and the effect of cavitation occurrence to bearing characteristics for oil-lubricated spiral-grooved journal bearings. Then, the authors reported, for an example, that the cavitation region maps obtained by a visualization experiment with transparent sleeve agree comparatively well with theoretical ones calculated with the equivalent flow model ${ }^{(6),(7)}$. These studies, however, concerns only with 'static' bearing characteristics. To aim to apply the spiral-grooved bearings to the spindle of small-sized precision instruments, the grasp of the 'dynamic' characteristics of the bearing with considering cavitation occurrence should be necessarily required.

In this paper, then, the dynamic bearing characteristics of oil-lubricated spiral-grooved journal bearings with considering the cavitation occurrence are theoretically investigated. The dynamic bearing characteristics, those are dynamic bearing stiffnesses and damping coefficients, are obtained by applying the perturbation scheme to the modified Reynolds equation formulated by cooperating with the equivalent flow model. The stable operating limits against Half-Frequency Whirl (HFW), which essentially occurs in the fluid film bearings, are examined as well.

\section{Nomenclature}

$c_{r}$ : Bearing clearance

$k$ : Cavitation coefficient

$p_{a}:$ Atmospheric pressure

$r_{0}:$ Bearing radius

$B_{X X}, B_{Y X}$ : Dimensionless damping coefficients

$H$ : Dimensionless local film thickness $\left(=h / c_{r}\right)$

$K_{X X}, K_{Y X}$ : Dimensionless bearing stiffnesses

$L$ : Dimensionless bearing length $\left(=l / r_{0}\right)$

$P$ : Dimensionless local pressure $\left(=p / p_{a}\right)$

$P_{e}$ : Dimensionless threshold pressure for cavitation occurrence $\left(=p_{e} / p_{a}\right)$

$\beta$ : Groove angle

$\delta$ : Dimensionless groove depth $\left(=\bar{\delta} / c_{r}\right)$

$\kappa:$ Local volumetric ratio of liquid to the volume of bearing gap

$\lambda$ : Bearing number

$\mu$ : Viscosity of liquid

$\bar{\mu}$ : Equivalent viscosity of cavitated fluid

$v$ : Angular velocity for squeeze motion

$\rho$ : Density of liquid $\bar{\rho}$ : Equivalent density of cavitated fluid

$\sigma:$ Squeeze number

$\tau$ : Dimensionless time $(=v t)$

$\omega$ : Angular velocity of shaft rotation

$\Omega:$ Whirl ratio $(=v / \omega)$

\section{Basic Equations and Method of Analysis}

\subsection{Bearing model for analysis}

The schematic diagram of a spiral-grooved journal bearing for the study is shown in Fig. 1. As a basic bearing model, the authors suppose that it has only one herringbone-grooved region and that the extending plain regions are arranged at both sides of the grooved region. It is noted that eccentricity ratio of the shaft, $\varepsilon$, is defined as a ratio of a parallel displacement of the shaft to the bearing clearance at the central grooved region $(0 \leq \varepsilon \leq 1)$, and the $X$-axis is set at the direction of shaft displacement.

\subsection{Basic equations}

3.2.1 Applications of equivalent flow model to Reynolds equation 'Equivalent flow model' was proposed by Ikeuchi, K. as a basic model for analysis with considering the effect of cavitation occurrence ${ }^{(5)}$. He applied the model to an analysis of plain journal bearing, and discussed the bearing characteristics with considering the cavitation effect. The model is based on the following assumptions:

- Bearing clearance is so small that a cavitated fluid flow is stably in a form of two-phase laminar flow consisted of liquid and gaseous (air) portions.

- Liquid is incompressible, and its density is constant. Density and viscosity of gaseous (air) portion in cavitation region are much smaller than those of liquid, while fluidity of gas (air) is much larger than that of the liquid.

- Cavitations occur in the regions under atmospheric pressure, that is, threshold pressure for cavitation occurrence is assumed that $p_{e}=p_{a}$.

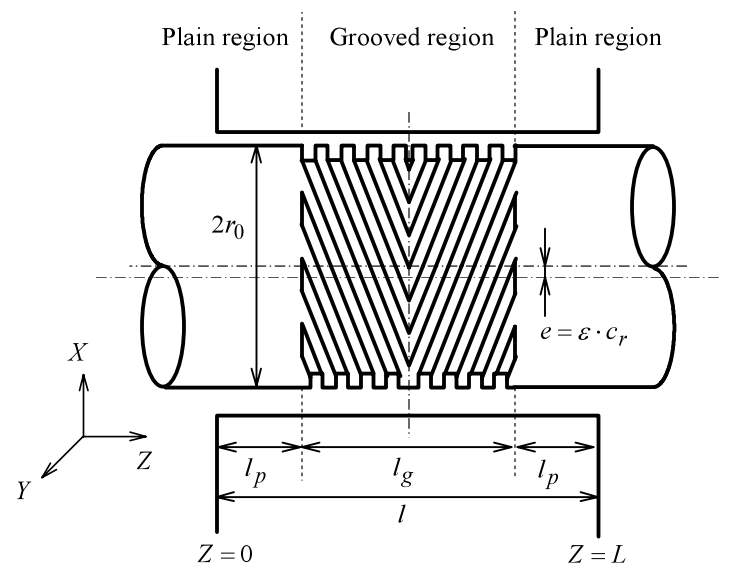

Fig. 1 Schematic diagram of a spiral-grooved journal bearing 
Here we introduce a local parameter $\kappa$, which expresses a volumetric ratio of liquid to the volume of the bearing gap. $\kappa$ changes from 0 to 1 ; where $\kappa=0$, the bearing clearance is filled with air, while the clearance is filled with liquid where $\kappa=1$. Density and viscosity of cavitated fluid is then assumed to be in proportion to $K$ as follows:

$$
\begin{aligned}
& \bar{\rho}=\kappa \rho \\
& \bar{\mu}=\kappa \mu
\end{aligned}
$$

where $\kappa$ is given as follows:

$$
\kappa=1+k\left(P-P_{e}\right)
$$

$P_{e}$ represents dimensionless threshold pressure for cavitation occurrence. In this study, $P_{e}=1$ by following the above third assumption. $k$, a parameter called 'cavitation coefficient', is defined as follows:

$$
\begin{aligned}
& k=0 \quad\left(P \geq P_{e}\right) \\
& k=k_{\text {const. }} \quad\left(P<P_{e}\right)
\end{aligned}
$$

where $k_{\text {const. }}$ means a degree of change of volumetric ratio of liquid to the volume of the bearing gap at liquidcavitation interface. Note that it is desirable to make $k_{\text {const. }}$ as large as possible in the computational process to clearly distinguish between fully fluid film region and cavitated regions.

Thus we can obtain the following equation by substituting Eqs. (1) and (2) into Reynolds equation expressed by the boundary-fitted coordinate system (see Fig. 2$)^{(8)}$ :

$$
\begin{gathered}
-\frac{\partial}{\partial \xi} H^{3}(A P-D P)+\frac{\partial}{\partial \eta}\left\{H^{3}(B P-C P)+\kappa E H+\kappa F\right\} \\
-G \frac{\partial(\kappa H)}{\partial \tau}=0
\end{gathered}
$$

where

$$
\begin{aligned}
& A \equiv 1 / \sin \beta \cdot \partial / \partial \xi \\
& B \equiv 1 / \tan \beta \cdot \partial / \partial \xi \\
& C \equiv 1 / \sin \beta \cdot \partial / \partial \eta
\end{aligned}
$$
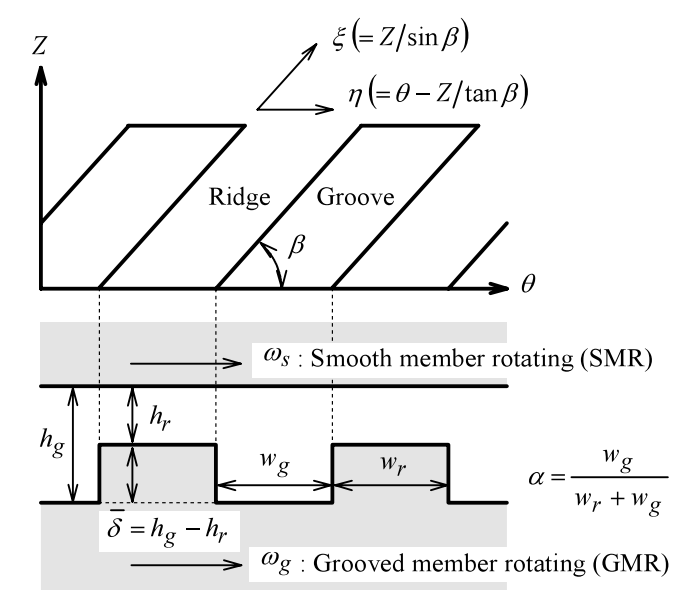

Fig. 2 Boundary-fitted coordinate system for spiral-grooved bearing

$$
\begin{aligned}
& D \equiv 1 / \tan \beta \cdot \partial / \partial \eta \\
& E \equiv \lambda \sin \beta \\
& G \equiv \sigma \sin \beta
\end{aligned}
$$

$F$ is separately defined according to the bearing system as follows:

For the case of Smooth Member Rotating (SMR) state,

$$
F=0
$$

while for the case of Grooved Member Rotating (GMR) state,

$$
\begin{array}{ll}
F=-2 \delta \lambda_{g} \sin \beta & \text { (on groove-ridge boundary) } \\
F=2 \delta \lambda_{g} \sin \beta & \text { (on ridge-groove boundary) } \\
F=0 & \text { (elsewhere) }
\end{array}
$$

$\lambda$ and $\sigma$ are bearing number and squeeze number, respectively, defined as follows:

For the case of SMR state,

$$
\lambda=\lambda_{s}=\frac{6 \mu \omega_{s}}{p_{a}} \cdot\left(\frac{r_{0}}{c_{r}}\right)^{2}
$$

while for the case of GMR state,

$$
\lambda=\lambda_{g}=\frac{6 \mu \omega_{g}}{p_{a}} \cdot\left(\frac{r_{0}}{c_{r}}\right)^{2}
$$

For the both cases,

$$
\sigma=\frac{12 \mu v}{p_{a}} \cdot\left(\frac{r_{0}}{c_{r}}\right)^{2}
$$

where $\omega_{s}$ and $\omega_{g}$ express the rotating angular velocities of the smooth member or the grooved member, respectively, according to SMR or GMR state, and $v$ does squeezing angular velocity.

The boundary conditions used are:

$$
\begin{aligned}
& \left.P\right|_{\theta=0}=\left.P\right|_{\theta=2 \pi} \\
& \left.P\right|_{Z=0}=\left.P\right|_{Z=L}=1
\end{aligned}
$$

Equation (12) represents periodic boundary condition in circumferential direction and (13) does longitudinal condition at both ends of the bearing.

3.2.2 Expansion of Reynolds equation by perturbation scheme Let us suppose a situation that the shaft (or relatively sleeve) oscillates around the equilibrium state with a small amplitude in $X$-direction. Then, local film thickness can be described with using the bearing clearance in equilibrium state, $H_{0}$, and amplitude of the oscillation, $\Delta H$, as follows:

$$
H=H_{0}+\Delta H \cos \theta \cdot e^{i \tau}
$$

It is also assumed that a consequent change of the pressure distribution in the bearing clearance can be expressed as:

$$
P=P_{0}+\Delta P e^{i \tau}
$$

where $\Delta P$ is complex amplitude of pressure oscillation. Then, we can get the following equation by picking out the 
first order incremental terms from Eq. (5) after substituting Eqs. (14) and (15) into Eq. (5):

$$
\begin{aligned}
- & \frac{\partial}{\partial \xi}\left\{H_{0}^{3}(A \Delta P-D \Delta P) \cdot e^{i \tau}\right. \\
& \left.+3 H_{0}^{2} \Delta H \cos \theta\left(A P_{0}-D P_{0}\right) \cdot e^{i \tau}\right\} \\
& +\frac{\partial}{\partial \eta}\left\{H_{0}^{3}(B \Delta P-C \Delta P) \cdot e^{i \tau}\right. \\
& \left.+3 H_{0}^{2} \Delta H \cos \theta\left(B P_{0}-C P_{0}\right) \cdot e^{i \tau}\right\} \\
& +\frac{\partial}{\partial \eta}\left[E \Delta H \cos \theta\left\{1+k\left(P_{0}-P_{e}\right)\right\} \cdot e^{i \tau}\right. \\
& \left.+E H_{0} k \Delta P e^{i \tau}+F k \Delta P e^{i \tau}\right] \\
& -i G \Delta H \cos \theta\left\{1+k\left(P_{0}-P_{e}\right)\right\} \cdot e^{i \tau}-i G H_{0} k \Delta P e^{i \tau}=0
\end{aligned}
$$

Solving the above equation, we will be able to obtain a complex amplitude of oscillation of pressure in the bearing clearance, $\Delta P$, under the supposed oscillating motion of a shaft (or relatively sleeve). If the complex amplitude $\Delta P$ is divided into real and imaginary parts as $P_{1}+i P_{2}$, dimensionless dynamic stiffnesses $K_{X X}, K_{Y X}$ and damping coefficients $B_{X X}, B_{Y X}$ are obtained as follows:

$$
\begin{aligned}
K_{X X} & =\iint P_{1} \cos \theta d \theta d Z /(2 L \Delta H) \\
K_{Y X} & =\iint P_{1} \sin \theta d \theta d Z /(2 L \Delta H) \\
B_{X X} & =\iint P_{2} \cos \theta d \theta d Z /(2 L \Delta H) \\
B_{Y X} & =\iint P_{2} \sin \theta d \theta d Z /(2 L \Delta H)
\end{aligned}
$$

It is noted that, as one can notice through the introduction of Eq. (16), the above procedures imply that the cavitation regions are also assumed to change in proportional with the perturbed shaft displacement.

\subsection{Computational schemes}

Discretization of Eqs. (5) and (16) by applying the concept of 'divergence formulation method' and application of the over relaxation scheme to solve the obtained simultaneous equations for the pressures on the discretized grid points lead to the numerical solutions of $P_{0}$ and $\Delta P$. At the computational process, the author has applied some useful techniques for more stable convergence as follows:

- At solving Eq. (5), the 'implicit scheme' is applied to $P$ appearing in Eq. (3).

- The 'windward stream scheme' is also applied to $P H$ terms appearing in $\kappa H$ term at both calculation processes of Eqs. (5) and (16).

For the numerical calculation, the authors prepare $240 \times 96$ grids in circumferential and axial directions, respectively. The calculation process by over relaxation scheme is judged to give a convergent solution if $\sum\left\{\left|P_{1}^{(n)}-P_{1}^{(n-1)}\right|+\left|P_{2}^{(n)}-P_{2}^{(n-1)}\right|\right\}<0.001$ is satisfied. $k_{\text {const. }}$ appearing in Eq. (4) is settled to be about 7 on a confirmation that the obtained solutions scarcely change even if $k_{\text {const. }}$ is set to be a larger value.

\section{Results of Stiffnesses and Damping Coefficients}

\subsection{Effect of squeeze number}

The specifications of the bearing supposed for the numerical calculation in this study is shown in Table 1 . In this study, bearing number $\lambda$ is set as 10 or 20. Obtained results of dimensionless stiffnesses and damping coefficients are respectively expressed in the forms of $K / \lambda$ and $B / \sigma$, because the values of $K / \lambda$ and $B / \sigma$ become constant against variations of $\lambda$ and $\sigma$, if the effect of cavitation occurrence is neglected.

The dimensionless dynamic stiffnesses and damping coefficients with changing squeeze number are shown in Figs. 3 and 4, respectively. The results with considering or neglecting cavitation occurrence are shown by closed marks and open marks, respectively, and circles represent the results for smooth member rotating (SMR) case and the squares are for groove member rotating (GMR) case.

In Figs. 3 and 4, we can see that $K_{X X}$ calculated with considering cavitation effect is larger than that with neglecting it, and that $K_{Y X}, B_{X X}$, and $B_{Y X}$ with considering it are smaller than those with neglecting it. These trends of $K_{X X}$ and $K_{Y X}$ can be explained, as follows: when cavitation occurrence is considered, pressure value in the divergent film region after the minimum bearing clearance point becomes to be almost atmospheric pressure level, while the pressure there remains at a negative level if cavitation occurrence is not considered. These differences in pressure level yield a larger $K_{X X}$ and a smaller $K_{Y X}$ value when cavitation occurrence is considered. Note that these tendencies are essentially the same as seen for the case of plain journal bearings. The trends of $B_{X X}$ and $B_{Y X}$ can be explained by the fact that the cavitation occurrence is likely to be induced around $\theta=0$ by reverse squeezing action, resulting in a decrease of total squeeze pressure if the cavitation occurrence is considered.

Now let us focus on the influence of the change of squeeze number to the bearing characteristics. Figures 3 and 4 show that the values of bearing characteristics, $K / \lambda$ and $B / \sigma$, remain to be constant against change of squeeze number if the cavitation occurrence is neglected. When

Table 1 Dimensions of analyzed bearing

\begin{tabular}{l|l}
\hline \hline Lengths & $\begin{array}{l}l_{g} / r_{0}=2.0 \\
l_{p} / r_{0}=0.4\end{array}$ \\
\hline Clearance ratio & $c_{r} / r_{0}=0.002$ \\
\hline Groove depth & $\bar{\delta} / c_{r}=1.1$ \\
\hline Ratio of groove width & $\alpha=0.5$ \\
\hline Groove angle & $\beta=33.0$ \\
\hline Number of grooves & $N=15$ \\
\hline
\end{tabular}



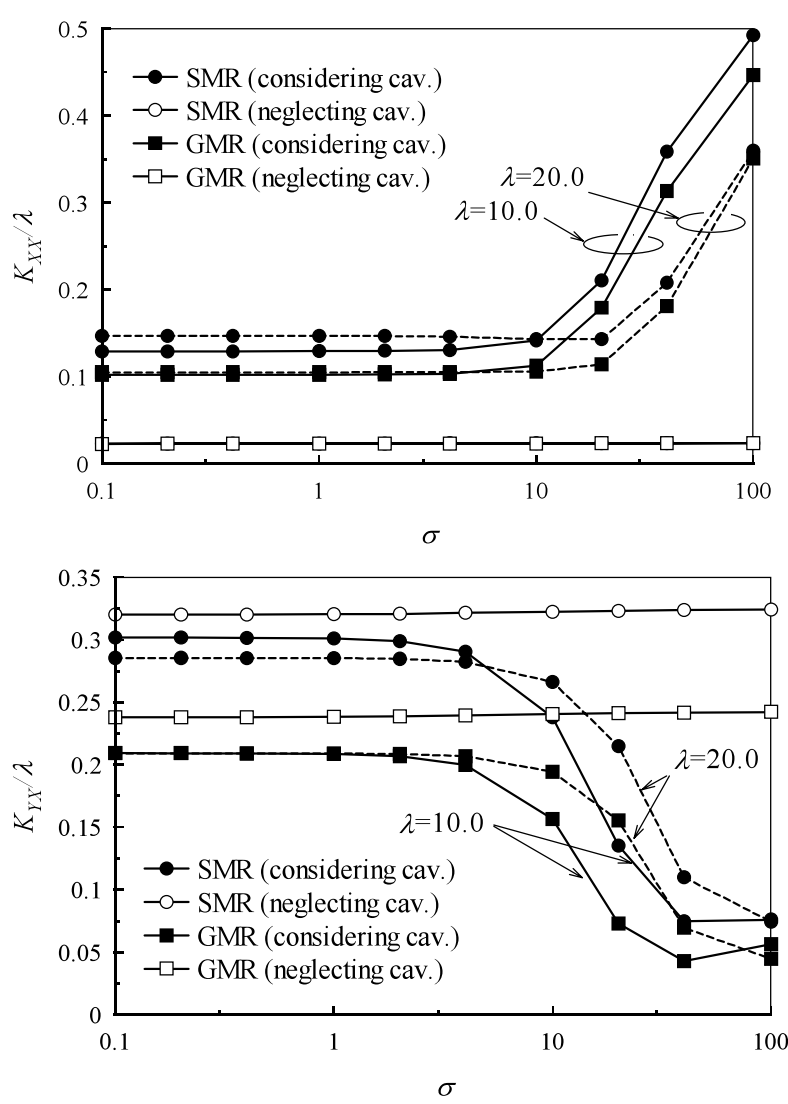

Fig. 3 Dynamic bearing stiffnesses versus squeeze number
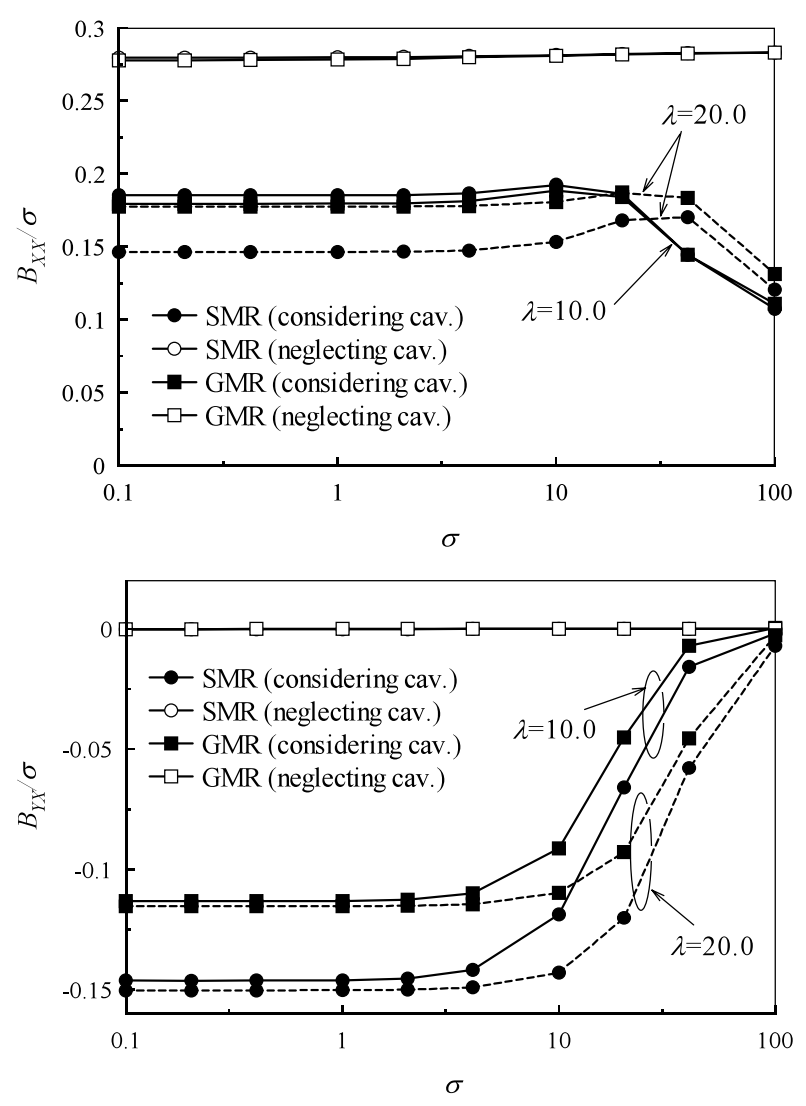

Fig. 4 Bearing damping coefficients versus squeeze number we consider the effect of cavitation occurrence, on the other hand, it can be noticed that these values considerably change especially when $\sigma$ exceeds a certain value; about $\sigma=10$ for the case of $\lambda=10$, and about $\sigma=20$ for the case of $\lambda=20$. It means that the dynamic characteristics are considerably influenced by squeeze number especially when whirl ratio $\Omega$ exceeds 0.5 , where the whirl ratio is defined as the ratio of the angular velocity of whirling motion of the rotor to the rotational velocity of the rotor as $\Omega=v / \omega=\sigma / 2 \lambda$. At the situation where the value of $\Omega$ is larger than 0.5 , the values of $K_{Y X}$ and $B_{Y X}$ become larger and $K_{Y X}$ and $B_{X X}$ smaller.

As to a difference of the rotational systems (that is, either SMR or GMR system), the figures show that $K_{X X}$ and $K_{Y X}$ in SMR system are larger and $B_{X X}$ and $B_{Y X}$ in SMR system are smaller than those in GMR system. This tendency can be seen for both cases with considering and neglecting cavitation occurrence.

\subsection{Effect of steady-state eccentricity}

The effect of steady-state eccentricity ratio of the rotor, $\varepsilon_{0}$, to the bearing characteristics are shown in Figs. 5 and 6 , where the former shows dimensionless bearing stiffnesses, while the latter damping coefficients. The condition supposed at calculations are such that the bearing number $\lambda=10$ and the squeeze number $\sigma=20$. The usage of the symbols and lines in the figures is the same as in
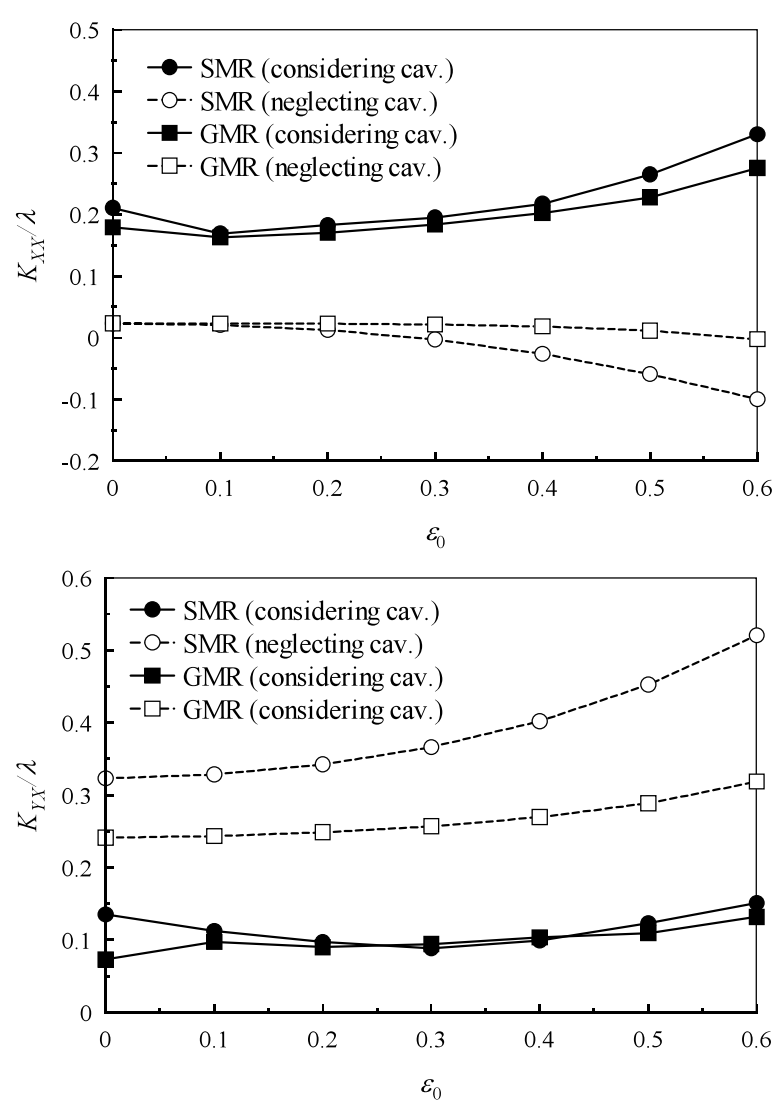

Fig. 5 Dynamic bearing stiffnesses versus eccentricity 

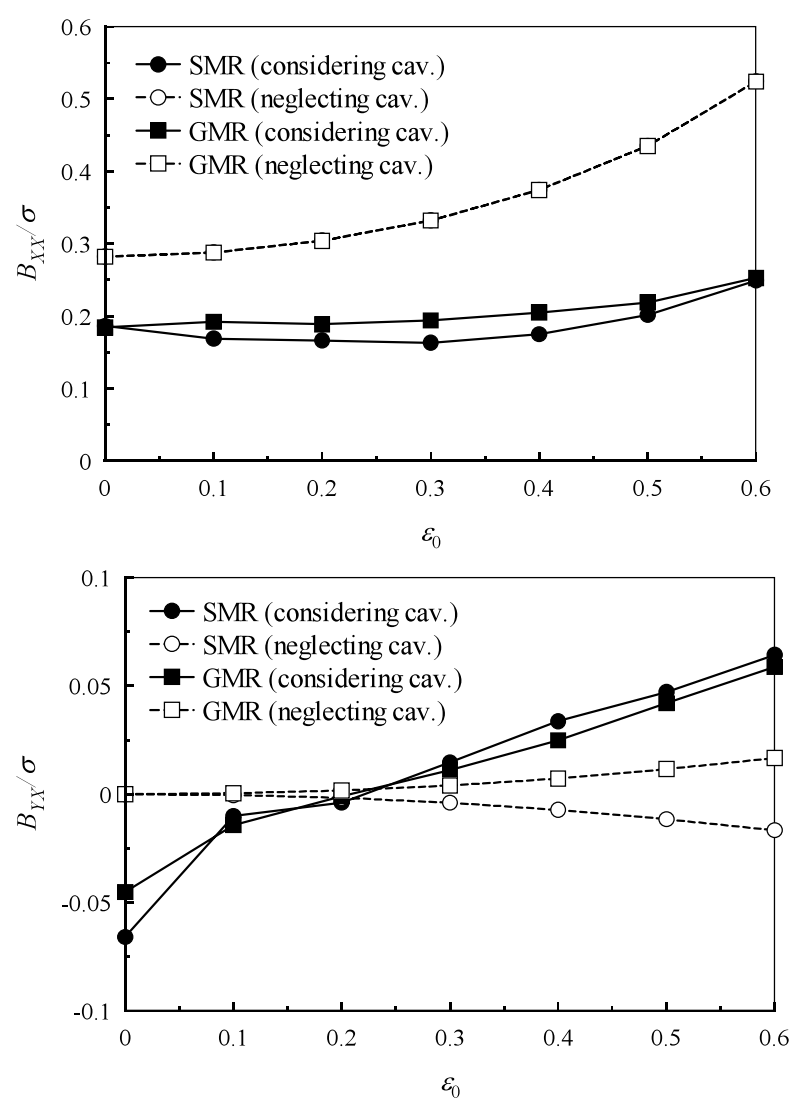

Fig. 6 Bearing damping coefficients versus eccentricity

Figs. 3 and 4.

These figures show that, if the cavitation occurrence is considered, all values of $K$ and $B$ increase in accordance with increase of $\varepsilon_{0}$. The effects of $\varepsilon_{0}$ is rather marked for the case with neglecting the occurrence of cavitation than that with considering it except for $B_{Y X}$. As a whole, however, it can be concluded that the eccentricity of the rotor does not affect so much the bearing characteristics though the cavitated area may become larger at a high eccentricity state.

\section{Discussions on Stable Operating Limits}

All of the hydrodynamically lubricated bearings essentially have a possibility to encounter a 'half-frequency whirl (HFW)', which is a kind of self-exciting rotor vibrations induced by the oil film force. It is also known that the condition if a bearing encounters HFW or not depends particularly on the mass of the rotor and the bearing number.

The stable operating limits of a rotor supported by spiral-grooved journal bearing against HFW in cylindrical mode will be investigated in this section.

\subsection{Fundamental equation for stable operating limits}

Applying Routh-Hurwitz criterion to the equation of motion of the shaft, we can obtain the equations for stable operating limit as follows in terms of the critical value of rotor mass and whirl ratio $\Omega^{(9),(10)}$ :

$$
\begin{aligned}
& M \cdot \lambda=\frac{36}{\Omega^{2}} \cdot \frac{K_{0} B_{0}+K_{1} B_{1}}{B_{0}} \\
& \Omega \equiv \frac{\sigma}{2 \lambda} \\
& =\frac{1}{2} \sqrt{\frac{\left(K_{0} B_{0}+K_{1} B_{1}\right)^{2}-2 K_{0} B_{0}\left(K_{0} B_{0}+K_{1} B_{1}\right)+B_{0}^{2}\left(K_{0}^{2}+K_{1}^{2}\right)}{B_{0}^{2}\left(B_{0}^{2}+B_{1}^{2}\right)}}
\end{aligned}
$$

where

$$
\begin{aligned}
& K_{0} \equiv K_{X X} / \lambda=K_{Y Y} / \lambda \\
& K_{1} \equiv K_{Y X} / \lambda=-K_{X Y} / \lambda \\
& B_{0} \equiv B_{X X} / \sigma=B_{Y Y} / \sigma \\
& B_{1} \equiv B_{Y X} / \sigma=-B_{X Y} / \sigma
\end{aligned}
$$

The former equation expresses the relation of the critical rotor mass for the stable operating limit and whirl ratio under the parallel mode HFW, while the latter does the whirl ratio observed when the condition just reaches the limit. $M$ in Eq. (21) is dimensionless critical mass of the rotor defined as follows:

$$
M=\frac{m}{l_{0} d_{0}} \cdot \frac{r_{0} p_{a}}{\mu^{2}} \cdot\left(\frac{r_{0}}{c_{r}}\right)^{5}
$$

The discussion developed in section 4 reveals that, if the effect of cavitation occurrence is considered, the values of dynamic bearing characteristics become variable with both of $\lambda$ and $\sigma$. It means that Eq. (22) is a 'non-linear' equation with respect to $\sigma$ under a given $\lambda$ value, resulting in a necessity of repeating process in calculation to obtain the value of $\sigma$ which satisfies Eq. (22). After the repetition process for obtaining $\sigma$ value, the critical mass value against the stable operation limit can be calculated from Eq. (21).

\subsection{Theoretical results}

Stable operating limit against parallel mode HFW of the bearing is shown in Fig. 7. The bearing dimensions for the study are listed in Table 1. Figure 7 (a) shows that the solutions under the condition of $\varepsilon_{0}=0.0$, while Fig. 7 (b) does under $\varepsilon_{0}=0.3$. Solid lines and dotted lines in the figures show, respectively, the results with considering and neglecting the cavitation occurrence.

Both figures indicate that, if we consider the effect of cavitation occurrence, the critical mass value of the rotor, which induces an unstable operation, becomes larger than that for the case with neglecting it, because the occurrence of cavitation makes $K_{X X}$ value larger.

It is noted in Fig. 7 (b) that we cannot find the result for the case of SMR system with neglecting the effect of cavitation occurrence. It is due to that, under such a situation, the stable operation condition is not obtained, that is, the rotor is calculated to be always unstable under any operating condition. This fact can be explained by referring to the results of Fig. 5: We can see in Fig. 5 that, as an 


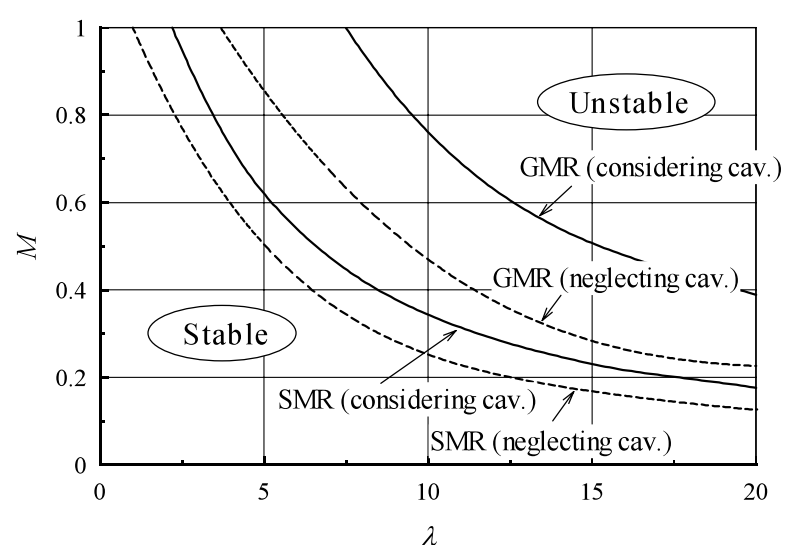

(a) Under the condition of $\varepsilon_{0}=0.0$

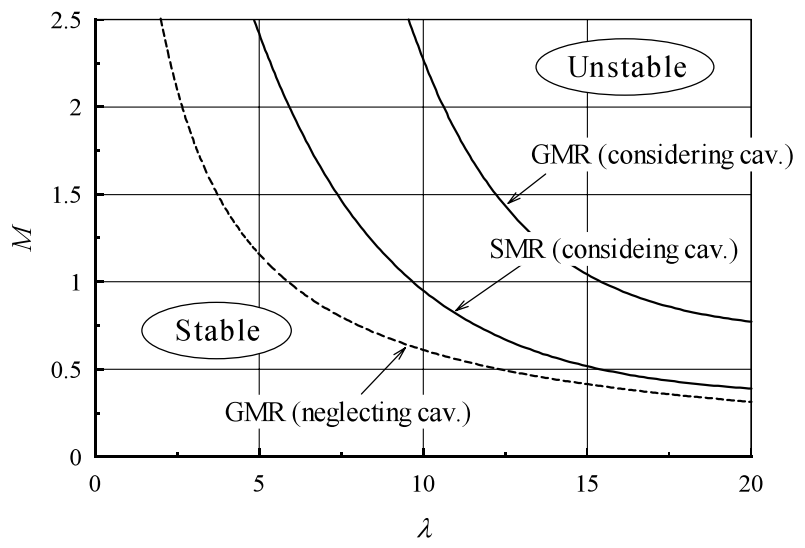

(b) Under the condition of $\varepsilon_{0}=0.3$

Fig. 7 Stability limits for parallel mode HFW

increase in steady-state eccentricity ratio, $K_{X X}$ value gradually decreases to be negative. The negative $K_{X X}$ means that the film force works not toward the opposite direction but toward the same direction of the rotor displacement, so that the rotor is forced to increase its displacement much more to be in unstable operation. However, it does not seem to be the fact from our engineering experiences. Thus we can conclude that the cavitation occurrence plays an important role at the practical situation. It can be said that we should consider the cavitation occurrence by an appropriate manner if we need to make a reliable evaluation of the bearing characteristics.

As to the effect of the rotating system of the bearing, these figures also show that GMR system is, in general, more stable than SMR system.

Table 2 shows the results of whirl ratio $\Omega$ of the selfexciting vibration of the rotor at the threshold condition. The whirl ratio is calculated to be $\Omega \approx 0.5$, which may be a circumferential evidence for concluding that the vibration is HFW.

\section{Conclusions}

The effects of cavitation occurrence to the dynamic bearing characteristics of spiral-grooved journal bearings are examined theoretically in this paper. The obtained re-
Table 2 Whirl ratios in parallel mode half-frequency whirl

\begin{tabular}{l|l|l}
\hline \hline$\varepsilon_{0}=0.0$ & SMR (considering cav.) & 0.56 \\
\hline & GMR (considering cav.) & 0.42 \\
\hline & SMR (neglecting cav.) & 0.58 \\
\hline & GMR (neglecting cav.) & 0.42 \\
\hline$\varepsilon_{0}=0.3$ & SMR (considering cav.) & 0.56 \\
\hline & GMR (considering cav.) & 0.40 \\
\hline & SMR (neglecting cav.) & 0.56 \\
\hline & GMR (neglecting cav.) & 0.38 \\
\hline
\end{tabular}

sults are summarized as follows:

- The Reynolds equation together with equivalent flow model is expanded by the perturbation scheme with respect to the journal eccentricity, so that the dynamic bearing characteristics such as the bearing stiffnesses and damping coefficients can be calculated from the obtained results of perturbed pressure distributions.

- If the cavitation occurrence is considered, the values of the direct bearing stiffness are calculated to be larger, while the ones of the cross-coupling stiffness and damping coefficient become smaller than those when it is neglected. It is explained by fact that the film pressure in divergent region in the bearing is lifted up by the occurrence of cavitation compared with otherwise case.

- Dynamic bearing characteristics calculated with cavitation occurrence are considerably influenced by squeeze number, especially when the whirl ratio exceeds 0.5. It suggests that a careful consideration about the whirling motion of the rotor is required for an exact evaluation of bearing characteristics.

- The stable operating limit of the bearing against self-exciting vibration is discussed, showing that critical mass value which induces unstable operation become larger if the cavitation occurrence is considered. It is also shown that the whirl ratio at the threshold condition is about 0.5 , which proves that the encountered vibration could be classified into half-frequency whirl.

- In some cases, the theoretical treatment without cavitation occurrence yields a result which is different from our engineering experiences: The rotor operation is calculated to be unstable when we suppose that the eccentricity ratio is 0.3 for SMR rotor system, for an example. It indicates that the cavitation occurrence is required to be considered with a sufficient care for estimating the exact bearing characteristics.

\section{Acknowledgement}

The authors wish to express a sincere gratitude to $\mathrm{Mr}$. N. Hishida of Spindle Device Laboratory, who often gave us many pieces of appropriate advice.

\section{References}

( 1 ) Ono, K., Needs and Trend of High Precision Rotation, J. of JSPE, (in Japanese), Vol.67, No.7 (2001), 
pp.1051-1054.

( 2 ) C.P.R., Ku., Dynamic Characteristics of Hard Disk Drive Spindle Motors - Comparison between Ball Bearings and Hydrodynamic Bearings -, Trans. ASME, J. of Tribology, Vol.118, No.2 (1996), pp.402406.

( 3 ) Zhu, J. and Ono, K., A Comparison Study on the Performance of Four Types of Oil Lubricated Hydrodynamic Thrust Bearings for Hard Disk Drive Spindles, Trans. ASME, J. of Tribology, Vol.121, No.1 (1999), pp.114-120.

( 4 ) Jang, G.H. and Kim, Y.J., Calculation of Dynamic Coefficients in Hydrodynamic Bearing Considering Five Degrees of Freedom for a General Rotor-Bearing System, Trans. ASME, J. of Tribology, Vol.121, No.3 (1999), pp.499-505.

( 5 ) Ikeuchi, K. and Mori, H., The Effect of Cavity Fluctuation on the Elastic and Damping of Journal Bearings, Trans. Jpn. Soc. Mech. Eng., (in Japanese), Vol.53, No.485, C (1987), pp.136-143.

( 6 ) Hirayama, T., Sakurai, T. and Yabe, H., A Theoretical Analysis Considering Cavitation Occurrence in OilLubricated Spiral-Grooved Journal Bearings with Ex- perimental Verification, Trans. ASME, J. of Tribology (2004), in press.

( 7 ) Hirayama, T. and Yabe, H., A Study on Operating Characteristics of Spiral Grooved Journal Bearing An Investigation of the Boundary Contour of GasLiquid Interface - , Proc. of Int. Tribol. Conf., Nagasaki, (2000), pp.1633-1638.

( 8 ) Kawabata, N., A Study on Numerical Analysis of Fluid Film Lubrication by the Boundary-Fitted Coordinates System (1st Report, Fundamental Equations of DF Method and the Case of Incompressible Lubrication), Trans. Jpn. Soc. Mech. Eng., (in Japanese), Vol.53, No.494, C (1987), pp.2155-2160.

( 9 ) Ichihara, J., The Theoretical Investigations for the Stability of Herring-Bone Grooved Gas Journal Bearings (1st Report, Conical Mode Analysis), Trans. Jpn. Soc. Mech. Eng., (in Japanese), Vol.54, No.506, C (1988), pp.2325-2333.

(10) Ichihara, J., The Theoretical Investigations for the Stability of Herring-Bone Grooved Gas Journal Bearings (2nd Report, Experimental Study), Trans. Jpn. Soc. Mech. Eng., (in Japanese), Vol.54, No.506, C (1988), pp.2334-2341. 